\title{
Changes in muscle activity during the flexion and extension phases of arm cycling as an effect of power output are muscle-specific
}

\author{
Carla P Chaytor ${ }^{1}$, Davis Forman ${ }^{1}$, Jeannette Byrne ${ }^{1}$, Angela Loucks-Atkinson ${ }^{1}$, Kevin E Power ${ }^{\text {Corresp. } 1}$ \\ ${ }^{1}$ Human Kinetics and Recreation, Memorial University of Newfoundland, St. John's, Newfoundland, Canada \\ Corresponding Author: Kevin E Power \\ Email address: kevin.power@mun.ca
}

Arm cycling is commonly used in rehabilitation settings for individuals with motor impairments in an attempt to facilitate neural plasticity, potentially leading to enhanced motor function in the affected limb(s). Studies examining the neural control of arm cycling, however, typically cycle using a set cadence and power output. Given the importance of motor output intensity, typically represented by the amplitude of electromyographic (EMG) activity, on neural excitability, surprisingly little is known about how arm muscle activity is modulated using relative workloads. Thus, the objective of this study was to characterize arm muscle activity during arm cycling at different relative workloads. Participants $(n=11)$ first completed a 10-second maximal arm ergometry sprint to determine peak power output (PPO) followed by 11 randomized trials of 20-second arm cycling bouts ranging from $5-50 \%$ of PPO ( $5 \%$ increments) and a standard $25 \mathrm{~W}$ workload. All submaximal trials were completed at $60 \mathrm{rpm}$. Integrated EMG amplitude (iEMG) was assessed from the biceps brachii, brachioradialis, triceps brachii, flexor carpi radialis, extensor carpi radialis and anterior deltoid of the dominant arm. Arm cycling was separated into two phases, flexion and extension, relative to the elbow joint for all comparisons. As expected, iEMG amplitude increased during both phases of cycling for all muscles examined. With the exception of the triceps brachii and extensor carpi radialis, iEMG amplitudes differed between the flexion and extension phases. Finally, there was a linear relationship between iEMG amplitude and the \%PPO for all muscles during both elbow flexion and extension. 
1 Changes in muscle activity during the flexion and extension phases of arm cycling as an

8 Carla P. Chaytor ${ }^{1}$, Davis A. Forman ${ }^{1}$, Jeannette Byrne ${ }^{1}$, Angela Loucks-Atkinson ${ }^{1}$ and Kevin E.

9 Power $^{1,2^{*}}$

\section{Corresponding Author}

14 Kevin Power, Professor

15 Human Neurophysiology Lab, ${ }^{1}$ School of Human Kinetics and Recreation and ${ }^{2}$ Biomedical

16 Sciences, Faculty of Medicine

17 Memorial University

18 St. John's, Newfoundland

19 Physical Education Building | PE 2022A

20 Email: kevin.power@mun.ca

23 Running Head: Arm muscle activity during arm cycling 


\section{Abstract}

Arm cycling is commonly used in rehabilitation settings for individuals with motor

27 impairments in an attempt to facilitate neural plasticity, potentially leading to enhanced motor

28 function in the affected $\operatorname{limb}(\mathrm{s})$. Studies examining the neural control of arm cycling, however,

29 typically cycle using a set cadence and power output. Given the importance of motor output 30 intensity, typically represented by the amplitude of electromyographic (EMG) activity, on neural

31 excitability, surprisingly little is known about how arm muscle activity is modulated using relative

32 workloads. Thus, the objective of this study was to characterize arm muscle activity during arm 33 cycling at different relative workloads. Participants $(n=11)$ first completed a 10 -second maximal 34 arm ergometry sprint to determine peak power output (PPO) followed by 11 randomized trials of 3520 -second arm cycling bouts ranging from $5-50 \%$ of PPO (5\% increments) and a standard $25 \mathrm{~W}$ workload. All submaximal trials were completed at $60 \mathrm{rpm}$. Integrated EMG amplitude (iEMG) was assessed from the biceps brachii, brachioradialis, triceps brachii, flexor carpi radialis, extensor carpi radialis and anterior deltoid of the dominant arm. Arm cycling was separated into two phases, flexion and extension, relative to the elbow joint for all comparisons. As expected, iEMG amplitude increased during both phases of cycling for all muscles examined. With the exception of the triceps brachii and extensor carpi radialis, iEMG amplitudes differed between the flexion and extension phases. Finally, there was a linear relationship between iEMG amplitude and the \%PPO for all muscles during both elbow flexion and extension. 


\section{Introduction}

49

50

51

52

53

54

55

56

57

58

59

60

61

62

63

64

65

66

67

Arm cycling, also referred to as arm crank ergometry, is commonly used as a means of exercise in rehabilitation programs for individuals living with upper and/or lower limb impairments following, for example, stroke or spinal cord injury. While the benefits of this type of exercise for the metabolic and cardiovascular systems are important, an additional aim when used in neurological populations is to maintain functional motor output or to induce neural plasticity (Kaupp et al. 2018; Klarner et al. 2016), potentially leading to a regain of motor output in the affected limb(s). Given the importance of arm cycling to rehabilitation and the knowledge that exercise-induced adaptations are often intensity-dependent, surprisingly little information is available regarding how arm cycling intensity influences the activation of the arm musculature.

It is well-known that as muscle contraction intensity increases, so too will muscle activity as assessed via surface EMG, at least up to a certain intensity. Using the gastrocnemius muscle, Lippold was amongst the first to demonstrate that force and EMG increased linearly during isometric contractions (Lippold 1952). Since that time, numerous studies have examined the EMGforce relationship in various muscles during isometric contractions, with examples of linear and non-linear relationships having been demonstrated (Woods \& Bigland-Ritchie 1983). Assessing the EMG-force relationship during dynamic muscle contractions is more challenging due to numerous physiological and non-physiological factors (Farina 2006). Despite these issues, linear relationships between peak velocity and acceleration with the EMG amplitude of the elbow extensors during a ballistic elbow extension has been shown (Aoki et al. 1986) with similar results reported in the elbow flexors (Barnes 1980).

The isometric and dynamic motor outputs that have been examined, however, are fundamentally different than locomotor outputs (examples include leg cycling and arm cycling) 
71 which are characterized by the bilateral, rhythmic, and alternating activation of antagonistic

72 motoneurone pools and are under different neural control than isometric contractions (Carroll et

73 al. 2006; Forman et al. 2014; Forman et al. 2016a; Forman et al. 2016b; Sidhu et al. 2012; Zehr \&

74 Duysens 2004). The activation of various muscles during leg cycling has been studied in detail,

75 with the influence of factors such as pedalling rate and workload having been examined (for

76 detailed review see Hug and Dorel, 2009). As with the results obtained using isometric and other

77 dynamic contractions, both linear (Bigland-Ritchie \& Woods 1974; Duchateau et al. 1986; Taylor

78 \& Bronks 1994) and non-linear relationships (Duchateau et al. 1986; Hug et al. 2006; Hug et al.

79 2003; Lucia et al. 1997) between changes in power output and EMG have been demonstrated.

80 Interestingly, by increasing the power output via increased load, Duchateau and colleagues showed

81 a linear EMG-power output relationship with the soleus and a non-linear relationship with the

82 gastrocnemius, suggesting that muscle-dependent differences may exist (Duchateau et al. 1986).

83 These EMG relationships are further complicated between cycling phases. During arm

84 cycling, data is frequently discussed in terms of the two main propulsion phases; the flexion phase,

85 which pulls the handle towards the individual (driven by the elbow flexors), and the extension

86 phase, which pushes the handle away (driven by the elbow extensors). Smith et al. (2008) was

87 some of the first work to show that muscle activity of upper-limb muscles was continuously

88 influenced throughout the crank cycle (see Figures 2A and 2B in Smith et al. (2008)). Recently,

89 we demonstrated that there are unique phase differences between the biceps and triceps brachii

90 during arm cycling (Forman et al. 2019; Forman et al. 2015). While the biceps brachii exhibits

91 cyclical muscle activity, with large bursts in the EMG signal during the flexion phase and almost

92 no activity in the extension phase, the triceps brachii demonstrates bursts of muscle activity in both

93 phases (see Figure 1 of Forman et al. 2015 and Figure 1B of Lockyer et al. 2018). From a motor 
94 control standpoint, this may indicate that greater co-contraction is needed during the flexion phase

95 to oppose the biceps brachii/stabilize the elbow joint. This also suggests that the elbow flexors and

96 extensors are driven by unique motor control strategies. However, these statements are limited in

97 that muscle activity from other upper-limb muscles have not been characterized between the two

98 phases of arm cycling. Additionally, the unique phase characteristics of the biceps and triceps

99 brachii were observed in studies utilizing just 25W (Forman et al. 2019; Forman et al. 2015). Not

100 only are these workloads low, they are also absolute power outputs, which likely induce a greater

101 variation of muscle activity between individuals than relative workloads. It is presently unclear if

102 these phase differences persist, or are perhaps modulated, at higher relative arm cycling intensities.

103 There are only three studies that have shown the influence of workload on EMG of the arm

104 musculature during cycling (Bernasconi et al. 2006; Hundza et al. 2012; Spence et al. 2016). While

105 EMG increased in each study with increased workload, as would be expected, specific information

106 such as flexion/extension phase-dependence or activation pattern was not provided (Bernasconi et

107 al. 2006; Hundza et al. 2012) and/or there were minimal workloads utilized (Spence et al. 2016).

108 The objective of the present study was to characterize arm muscle activity (i.e. iEMG amplitude)

109 at different relative workloads during two different phases of arm cycling, flexion and extension,

110 as defined by movement at the elbow. We hypothesized that constant cadence cycling at different

111 mechanical loads (i.e. different power outputs) would result in: 1) increased iEMG amplitude 2)

112 phase-dependent differences in iEMG amplitudes 3) a linear relationship between iEMG amplitude

113 and power output and 4) differences in iEMG amplitudes between an absolute 25W workload and

114 a low relative workload of just $5 \%$ of peak power output (PPO).

115

116 Methodology

Peer] reviewing PDF | (2020:04:48201:1:1:NEW 15 Jul 2020) 


\section{Ethical Approval}

118 The procedures of the experiment were verbally explained to each volunteer prior to the

119 start of the session. Once all questions were answered, written consent was obtained. This study

120 was conducted in accordance with the Helsinki declaration and approved by the Interdisciplinary

121 Committee on Ethics in Human Research at Memorial University of Newfoundland (ICEHR\#:

122 20150140-HK). Procedures were in accordance with the Tri-Council guidelines in Canada and 123 potential risks were fully disclosed to participants.

124

125 Participants

126 Eleven healthy individuals (six males and five females, $25.2 \pm 4.4$ years of age, $73.6 \pm$ 127 7.8kg, nine right-hand dominant, two left-hand dominant) were recruited for this study. 128 Participants had no known neurological impairments. Prior to the experiment, all participants 129 completed a Physical Activity Readiness Questionnaire (PAR-Q+) to screen for any 130 contraindications to exercise or physical activity and an Edinburgh Handedness Inventory 131 checklist to quantify hand dominance. Participants were required to refrain from any heavy 132 exercise, especially upper body exercise, 24 hours prior to the start of testing.

\section{Experimental Procedure}

Participants attended a familiarization session to practice arm cycling sprints that were

136 required during the experimental session to determine peak power output (PPO). This session was 137 followed by an experimental session with a minimum of $24 \mathrm{hrs}$ between. During the experimental 138 session participants first completed a 5-minute warm-up using a Monark cycle ergometer 139 (Ergomedic $894 \mathrm{E}$ ), with only the $1 \mathrm{~kg}$ weighted basket as resistance, at a self-selected pace. The 
140 ergometer was securely mounted to the top of a table and fitted with hand pedals. Following the

141 warm-up and a 5-minute rest break, participants performed a 10 second maximal arm ergometry

142 sprint using $5 \%$ of their body weight as the resistance to determine PPO. The Monark cycle

143 ergometer recorded power output at a sampling rate of $50 \mathrm{~Hz}$; the highest power output of a single

144 sample throughout the 10 second trial was deemed to be the individual's PPO. Results of this

145 cycling trial were then used to determine the relative intensity for all subsequent trials. Following

146 a minimum 10 minutes post-sprint rest period, participants were moved to a SCIFIT cycle

147 ergometer (model PRO2 Total Body) to perform arm cycling at 11 different intensities, 10 of which

148 were made relative to the PPO and one which was done at $25 \mathrm{~W}$. The $25 \mathrm{~W}$ condition was constant

149 for all participants, given that $25 \mathrm{~W}$ is a common workload used during arm cycling studies (Bressel

150 et al. 2001; Forman et al. 2014). The remaining 10 trials were randomized and performed at relative

151 intensities ranging from 5-50\% of the PPO. For all trials participants cycled at a constant cadence

152 of $60 \mathrm{rpm}$ for 20 seconds.

153

154

155

156

Experimental Set-up

157 Participants were seated upright at a comfortable distance from the hand pedals, so that

158 during cycling, there was no reaching or variation in trunk posture (Fig. 1). To further ensure that

159 posture was maintained throughout all trials, each participant was strapped securely to the

160 ergometer seat with straps placed over the shoulders and across the chest. Movement of the

161 shoulders and arms was not impeded. The hand pedals of the ergometer were fixed 180 degrees

162 out of phase and the seat height was adjusted so that the shoulders of each individual were 
163 approximately the same height as the centre of arm crank shaft. Participants gripped the ergometer

164 handles with the forearms in a pronated position.

165 Cycle crank positions were made relative to a clock face (12, 3, 6, and 9 o'clock, as viewed 166 from the right crank arm) with the "top dead centre" position of the crank arm defined as 12 o'clock 167 and "bottom dead centre" as 6 o'clock, which is identical to previous investigations (Carroll et al. 168 2006; Klimstra et al. 2011; Nippard et al. 2020; Power et al. 2018; Zehr et al. 2004; Zehr \& Chua 169 2000). The biceps brachii and triceps brachii were the main muscles of interest, thus the 170 terminology used to describe the cycling movement is based on the position of the dominant elbow 171 joint. Elbow flexion was defined as the movement from 3 to 9 o'clock, while the hand was moving 172 toward the body. Elbow extension was defined as the movement from 9 to 3 o'clock, while the 173 hand was moving away from the body. There were magnets positioned at 3 o'clock and 9 o'clock 174 on the SciFit Bicycle in order to enable crank position to be tracked during cycling. When the 175 crank passed the magnets at the 3 o'clock and the 9 o'clock positions, a 5 volt pulse was sent from 176 the SciFit Bicycle to the data collection software. This pulse was recorded and used to track crank 177 position through-out all cycling trials.

178

179

180

181

182

183

184 185

\section{Electromyography Recording}

EMG of the biceps brachii, lateral head of the triceps brachii, anterior deltoid, brachioradialis, flexor carpi radialis (FCR), and extensor carpi radialis (ECR) of the dominant arm were recorded using pairs of surface electrodes (Medi-Trace 130 ECG conductive adhesive electrodes). The inter-electrode distance was $2 \mathrm{~cm}$ and all electrodes were aligned to fiber direction 
186 of the target muscles. A ground electrode was placed on the lateral epicondyle. Prior to electrode

187 placement the skin was thoroughly prepared by shaving any hair and the removal of dead epithelial 188 cells (using abrasive paper) followed by sanitization with an isopropyl alcohol swab. Muscle 189 activation data was collected at $2000 \mathrm{~Hz}$ using the BIOPAC MP-100 data acquisition system with 190 Acknowledge 4 software and an EMG100C differential amplifier (CMRR 110dB (50/60Hz), input 191 impedance $2 \mathrm{M} \Omega$, bandpass filter $10 \mathrm{~Hz}-500 \mathrm{~Hz}$ ). Data obtained during the experiment were 192 analyzed offline using code written in Visual Basic.

193

194

Data analysis

The EMG data were amplitude normalized by dividing the raw EMG during cycling by the 196 muscle specific maximum EMG from the 10-second maximal arm ergometry sprint. The maximum EMG amplitude was determined using a 100ms RMS moving window (as per Burden and Bartlett (1999)) to process the raw EMG from each muscle over the duration of the 10-second 199 sprint. The resulting smoothed signal was examined to determine the peak EMG for each muscle, 200 which was then used to normalize the amplitude of all sub-maximal cycling trials.

201

The submaximal cycling trials were then analyzed by examining the middle 10 seconds of 202 data from each trial. These 10 seconds of data were divided in to sections that represented one 203 complete revolution of the crank handle (from 3 o'clock to 3 o'clock). Each revolution was further 204 broken down in to an elbow flexion phase (from 3 o'clock to 9 o'clock) and an elbow extension 205 phase (from 9 o'clock to 3 o'clock). This was done using the magnet signal described above. For 206 most individuals, a total of 10 revolutions were completed during the 10 seconds of cycling. Once 207 the data was windowed, integrated EMG (iEMG) was calculated for the following time periods: 
208 the full revolution, the flexion phase and the extension phase. Trapezoid rule was used for these 209 calculations.

210 To assist with the visual presentation of the data, linear envelope, ensemble average EMG was

211 calculated for each arm cycling intensity. This was done using the following steps:

212

213

214

215

216

217

218

219

220

221

222

223

224

225

226

227

228

229

230
- Raw, amplitude normalized and windowed EMG was full wave rectified and low pass filtered at $10 \mathrm{~Hz}$ using a fourth order dual-pass butterworth filter. Data from each of the complete revolutions were then rubberbanded to normalize it to time. One revolution was considered $100 \%$ of the whole cycle with the time period from 3-9 being fit to the first $50 \%$ (flexion) of the rubberbanded signal and 9-3 to the last 50\% (extension).

- These rubberbanded trials were then ensemble averaged across all trials for each intensity. The end result was an average linear envelope for each muscle at each intensity.

\section{Statistics}

All statistical analysis was performed using IBM's SPSS Statistics Version 23. Assumptions of sphericity were tested using the Mauchley test, and if violated, the GreenhouseGeisser estimates of sphericity correction was applied to the degrees of freedom. The data were normally distributed as determined using the Kolomogorov-Smirnov normality test. Separate twoway (PHASE x INTENSITY) repeated-measures ANOVAs were used to assess the iEMG of each muscle ( $\mathrm{n}=11$ for biceps and triceps brachii and $\mathrm{n}=5$ for the remaining muscles) during two phases (flexion and extension) and 11 different workloads (25W and percentages of PPO). To determine whether the relationship between iEMG and intensity was best described as linear during both phases of arm cycling, a series of twelve repeated-measures one-way ANOVAs were conducted for each muscle examined using Polynomial Contrasts (i.e., linear, quadratic or cubic). Trends 
231 were determined by examining the F-values of each of the 3 models as well as the observed power.

232 All statistics were run on group data and a significance level of $p<.05$ was used. All data are 233 reported in text as means $\pm \mathrm{SD}$ and illustrated as means $\pm \mathrm{SE}$ in figures for clarity.

234

235

236

237

238

239

240

241

242

243

244

245

246

247

248

249

250

251

252

253

\section{Results}

EMG activity patterns of arm muscles during arm cycling

The EMG activity (i.e. linear envelope ensemble averaged EMG) for each of the arm muscles recorded during arm cycling are plotted in Figure 2. In this figure we have omitted EMG recorded from 5 of the 11 intensities (i.e. 5, 15, 25, 35 and 45\% of PPO) for figure clarity. There are several distinct qualitative features worth noting. First, the elbow flexor muscles, biceps brachii and brachioradialis are very active during elbow flexion (3 to 9 o'clock), while they are virtually silent during elbow extension (9 to 3 o'clock; Figs $2 \mathrm{~A}$ and B). The triceps brachii appears to be highly active during the extension phase, however, as opposed to the biceps brachii there is more of a biphasic activation pattern, with the muscle also being active during the flexion phase (Fig. 2C). FCR also appears to be biphasically active while ECR appears to have a peak activation occurring at roughly the 9 o'clock position, corresponding to the end of flexion/beginning of extension (Figs. 2D and E, respectively). The anterior deltoid is more active during the elbow extension phase (Fig. 2F), though it begins to activate during the latter portion of elbow flexion (i.e. approximately 6 o'clock). It is also clear that as the intensity of cycling increased so too did the EMG activation level in each muscle.

Intensity- and phase-dependent effects on iEMG of recorded muscles during arm cycling Table 1 summarizes the findings for each of the muscles recorded. All muscles demonstrated a significant main effect of INTENSITY on iEMG. With the exception of the triceps 
254 brachii and ECR, muscles also demonstrated significant main effects for PHASE (i.e. elbow

255 flexion vs extension) and an interaction effect between INTENSITY and PHASE.

256

257 Biceps brachii. A significant INTERACTION effect was shown with iEMG at each cycling 258 intensity being significantly different between flexion and extension; flexion had higher iEMG at 259 all levels of intensity compared to extension. During flexion, iEMG at $25 \mathrm{~W}$ was not statistically 260 different than 5\% PPO, though there was a trend for higher activation during the 5\% PPO cycling $261(p=0.052)$. The iEMG at subsequent ascending workloads significantly increased from 5 to $35 \%$ 262 PPO. Non-significant increases in iEMG occurred between 35 to 50\% PPO (i.e. iEMG recorded 263 at $35,40,45$ and 50\% PPO were not significantly different from each other though they were 264 greater than all intensities $\leq 30 \% \mathrm{PPO}$ ). During extension, changes in iEMG were similar to that 265 during flexion, with iEMG at 25W and 5\% PPO being not statistically different and subsequent 266 workloads in ascending order significantly increased from 5 to $35 \%$ PPO. Non-significant 267 increases in iEMG occurred between 35 to 50\% PPO (i.e. iEMG recorded at 35, 40, 45 and 50\% 268 PPO were not significantly different from each other though they were greater than all intensities $269 \leq 30 \%$ PPO).

270

271 Brachioradialis. A significant INTERACTION effect was shown with iEMG at each cycling 272 intensity being significantly different between flexion and extension; flexion had higher iEMG at 273 all levels of intensity compared to extension. During flexion, iEMG in the brachioradialis could 274 be grouped into three 'blocks' (block 1, 25W to 10\% PPO; block 2, 20 to $30 \%$ PPO; block 3, 40 275 to $50 \%$ PPO) and two stand-alone intensities (15 and 35\% PPO). The iEMG recorded within each 276 block were not different from each other but were different from the other blocks and also the 
277 stand-alone intensities. During extension, there were no differences between $25 \mathrm{~W}, 5$ and $10 \%$ PPO.

278 The iEMG during subsequent workloads significantly increased from 15 to 35\% PPO. Similar to

279 the biceps brachii, non-significant increases in iEMG occurred between 35 to 50\% PPO (i.e. iEMG

280 recorded at 35, 40, 45 and 50\% PPO were not significantly different from each other though they

281 were greater than all intensities $\leq 30 \%$ PPO).

282

283 Triceps brachii. There was a significant main effect for INTENSITY. With the data collapsed for

284 PHASE, a similar finding to that of the biceps brachii was revealed. Specifically, iEMG at $25 \mathrm{~W}$

285 was not statistically different than 5\% PPO but iEMG in subsequent workloads showed significant

286 increases from 5 to $35 \%$ PPO (i.e. iEMG recorded at 35, 40, 45 and 50\% PPO were not

287 significantly different from each other though they were greater than all intensities $\leq 30 \% \mathrm{PPO}$ ).

288

289 FCR. A significant INTERACTION effect was shown for iEMG, with each cycling intensity being 290 significantly different between flexion and extension, with the exception of the 15 and $50 \%$ PPO $291(\mathrm{p}=.074$ and $\mathrm{p}=.124$, respectively) intensities; flexion had higher iEMG at all other levels of 292 intensity compared to extension. Within both flexion and extension, iEMG at 25W, 5 and 10\% 293 PPO were not statistically different from one another. Subsequent workloads in ascending order 294 did not significantly increase from 10 to $50 \%$ PPO.

295

296 ECR. There was a significant main effect for INTENSITY (see Table 1) but not PHASE, thus data 297 was collapsed to examine the general influence of cycling INTENSITY on iEMG. There were no 298 significant differences in iEMG between 25W, 5 and 10\% PPO. Beginning at 15\% PPO iEMG 
299 significantly increased with subsequent increases in PPO up to and including 30\% PPO. iEMG 300 from 30 to 50\% PPO were not statistically different from each other.

301

302 Anterior Deltoid. During flexion, iEMG at 25W, 5 and 10\% PPO were not statistically different

303 from one another. Subsequent workloads in ascending order significantly increased from 10 to $30425 \%$ PPO. Non-significant increases in iEMG occurred from 25 to 50\% PPO (i.e. iEMG recorded 305 at 25, 30, 35, 40, 45 and 50\% PPO were not significantly different from each other though they 306 were greater than all intensities $\leq 20 \%$ PPO). During extension, iEMG at 25W, 5 and $10 \%$ PPO 307 were not statistically different from one another. Subsequent workloads in ascending order 308 significantly increased between 10 and 20\% PPO (i.e. 20>15>10). Non-significant increases in 309 iEMG occurred from 20 to 50\% PPO (i.e. iEMG recorded at 20, 25, 30, 35, 40, 45 and 50\% PPO 310 were not significantly different from each other though they were greater than all intensities $\leq 20 \%$ 311 PPO). As for the INTERACTION effect, iEMG at each cycling intensity was significantly 312 different between flexion and extension, with the exception of the $5 \%$ PPO $(\mathrm{p}=.058)$; extension 313 had higher iEMG at all levels of intensity compared to flexion.

314

The EMG-power output relationship during arm cycling is linear

Figures 3 (A-F) shows that the relationship between iEMG and workload was linear for all

317 muscles examined during both the flexion and extension phases of arm cycling, with details

318 provided in Table 2. We also compared the slopes of the linear relationships between flexion and 319 extension for each muscle using a paired t-test to assess if the gain in iEMG was different between 320 phases within a muscle. The slope was significantly different between flexion and extension for 
321 each muscle examined $(\mathrm{p}<0.001$ for biceps brachii, brachioradialis, FCR and anteriod deltoid; $\mathrm{p}$

$322=0.003$ for triceps brachii) with the exception of ECR $(p=0.07)$.

323

324 Discussion

325 This is the first study to characterize the flexion/extension specificity of arm muscle 326 activity during arm cycling over a wide range of power outputs. To do so we divided the arm 327 cycling motor output into two phases, flexion and extension, made relative to the elbow joint. 328 Using this criteria the most important results in the present study are: 1) arm muscle activity during 329 arm cycling at different relative PPOs was quantified, 2) iEMG amplitude increased significantly 330 with increased power outputs, 3) iEMG differed between the flexion and extension phases in all 331 muscles except the triceps brachii and ECR, and 4) a linear iEMG-power output relationship for 332 each of the muscles examined.

333

iEMG amplitudes during arm cycling

The basic pattern of arm muscle activity during arm cycling has been previously described 336 (Bressel et al. 2001; Zehr \& Chua 2000) but few studies have examined the influence of workload 337 on EMG of the arm muscles during arm cycling (Bernasconi et al. 2006; Hundza et al. 2012; Smith 338 et al. 2008; Spence et al. 2016) . Bernasconi et al. (2006) reported an increase in EMG with 339 increased workload in each of the muscles examined (biceps brachii, triceps brachii, anterior 340 deltoid, and infraspinatus) during an arm cycling $\mathrm{VO}_{2}$ max test. Their objective, however, was not 341 to give a detailed description of muscle activation levels as arm cycling intensity increased. For

342 example, they did not examine EMG during the two propulsive phases of arm cycling, nor did they

343 show representative traces of EMG activity. Similar findings were reported by Hundza et al. 
344 (2012), (i.e. increased EMG with increased arm cycling workloads). As was the case in the

345 Bernasconi report, however, they did not assess the phase-dependence or pattern of arm muscle

346 EMG, as that was not their intent. Smith et al. (2008) also demonstrated that muscle activity

347 increased as power output increased from 50 to $100 \mathrm{~W}$. However, asynchronous versus

348 synchronous cycling was the primary focus of this study, and with only two absolute workloads,

349 complete details into a power and muscle activity relationship were not elucidated. Finally, a recent

350 study from our lab examined corticospinal excitability to the biceps and triceps brachii during arm

351 cycling at two different workloads (Spence et al. 2016). In that study, however, only the biceps

352 and triceps brachii EMG were reported at two different workloads (5 and 15\% PPO) with EMG

353 recordings made at mid-elbow flexion and extension as previously discussed.

354 The present work, however, is the first to show patterns of arm muscle activity over a wide

355 range of power outputs during arm cycling (Fig. 2). Similar to previous work (Zehr \& Chua 2000)

356 reciprocal activation of functional antagonists was observed between muscles acting at the wrist

357 (FCR and ECR; Figs 2D and E, respectively) and to a certain degree at the elbow (i.e. during elbow

358 extension but not flexion; see biceps and triceps brachii traces in Figs 2A and C). Given our

359 laboratory's interest in the neural control of the biceps and triceps brachii musculature during arm

360 cycling (Copithorne et al. 2015; Forman et al. 2014; Forman et al. 2015; Forman et al. 2016a;

361 Forman et al. 2016b; Power \& Copithorne 2013; Spence et al. 2016), we were particularly

362 interested in the phase- and workload-dependent changes in those muscles. Interestingly, we show

363 that the elbow flexors (i.e. biceps brachii and brachioradialis) demonstrated strong phase-

364 dependence in EMG whereas the triceps brachii did not (compare Figs. 2A and B with C). This

365 finding is partially a function of the how we defined flexion and extension and thus during which

366 portions of the arm cycling revolution measurements were made. In our previous work, for 
367 example, we assessed EMG amplitude at mid-elbow flexion (6 o'clock) and mid-elbow extension

368 (12 o'clock) (Forman et al. 2014; Forman et al. 2016a; Forman et al. 2016b). These points in time

369 during a full cycle represent very different EMG activity levels than those averaged over half a

370 revolution as was done in the present study (see Fig. 2 A and C) and closely align with peak

371 activation in the biceps ( $\sim 5-6$ o'clock) and triceps brachii ( $\sim 10-11$ o'clock). In the present study

372 we separated and assessed iEMG activity during two phases, flexion (3 to $9 \mathrm{o}$ 'clock) and extension

373 (9 to 3 o'clock) relative to the elbow joint and not at specific points of the cycle.

374 Why do the biceps and triceps brachii demonstrate different phase-dependence

375 characteristics? The biceps brachii is bi-articular (i.e. crossing two joints - elbow and shoulder)

376 and contributes to elbow flexion. Thus, as expected, the biceps brachii was highly active during

377 elbow flexion and relatively inactive during elbow extension. The long head of the triceps brachii

378 is bi-articular (i.e. originates from the infraglenoid tubercle of the scapular, lateral and medial head

379 from the humerus), while the medial and lateral heads of the triceps brachii are mono-articular

380 activing only at the elbow. Because we recorded from the lateral head of the triceps brachii it was

381 surprising to find that its activity level was not different between the elbow flexion and extension

382 phases of arm cycling given its role in elbow extension. Though not assessed in the same manner

383 (i.e. elbow flexion vs extension), the triceps brachii muscle appears to demonstrate a stronger

384 phase-dependence in the study by Zehr and Chua (2000), though the head of the triceps from which

385 the recordings were made is not stated in that manuscript (Zehr and Chua, 2000). There are several

386 potential explanations for our present finding. First, arm cycling was performed with the hand in

387 a pronated position. Though forces at the hand pedal were not assessed, it is likely that during arm

388 cycling the elbow extensors are active during elbow flexion in an attempt to push the hand down

389 on the pedal to maintain a constant grip. Thus, the lateral head of the triceps may act as an extensor 
390 during elbow extension, while during elbow flexion, it may act to stabilize the hand. Second,

391 during elbow flexion the long head of the triceps acts as a prime mover to extend the shoulder (in

392 addition to the rear deltoid and latissimus dorsi). Because its primary role in the flexion phase of

393 arm cycling is likely shoulder extension, its capacity to stabilize the elbow may be limited.

394 Therefore, it is possible that the lateral and medial heads of the triceps brachii assume the role of

395 elbow stabilization, resulting in elevated iEMG in the flexion phase. A switch to a larger role

396 during elbow extension for the lateral head of the triceps may occur as the workload increases as

397 suggested via the higher gain in the iEMG-power output relationship, specifically at higher

398 workloads (see Figure 3C). Finally, activation of the lateral head of the triceps brachii may be

399 partially due to task novelty, resulting in unnecessary co-contraction. Recent work showed a

400 similar bi-phasic activation pattern of the triceps brachii that was abolished following arm cycling

401 training in persons with spinal cord injury (i.e. the triceps brachii activity was absent during

402 flexion) (Brousseau 2016), suggesting that a learning response may occur.

403

404 iEMG/power-output is linear for arm muscles during arm cycling

405 As expected, muscle activation levels (i.e. iEMG amplitudes) generally increased as arm

406 cycling workload increased for all muscles examined (see Table 1 and Fig. 3). The increases in

407 iEMG amplitudes reflect the increased recruitment and firing frequency of the motor units from

408 which we recorded. Based on the size principle (Adrian \& Bronk 1929; Henneman 1957) as the

409 cycling intensity increased, additional motor units, including larger, faster motor units, would be

410 recruited to assist with force production resulting in an increase in the iEMG amplitude. While the

411 iEMG-power output relationship was linear for each muscle, there are some points that should be

412 mentioned. It was noted that iEMG amplitudes were not significantly different when comparisons 
413 are made within the lowest (generally $25 \mathrm{~W}$ vs $5 \%$ PPO) and highest (generally $35-50 \%$ PPO)

414 cycling intensities for several muscles (see Results). While the lack of difference at the lower

415 intensities may simply be due to the fact that the cycling intensities were not high enough, it is

416 interesting that there was a general plateau of the iEMG at the higher power outputs given that the

417 highest workload was set at only $50 \%$ of PPO. This may be due to confounding variables related

418 to how the iEMG was normalized (see Methodological Considerations) or the result of torso or

419 lower extremity muscles making larger contributions to motor output execution at higher relative

420 PPO (Smith et al. 2008). Physiologically the plateau may be related to motoneurone pools being

421 near full recruitment and/or maximal firing rate. During isometric contractions, for example, spinal

422 motoneurone excitability as assessed via responsiveness to transmastoid electrical stimulation

423 plateaus at $\sim 50 \%$ of maximal voluntary effort (Pearcey et al. 2014). The continued increase in

424 power output likely involves changes in muscle co-ordination and/or muscle synergies (Blake \&

425 Wakeling 2015; Wakeling et al. 2010), keeping in mind that arm cycling is a bilateral motor output

426 that involves multiple muscles, many of which were not examined in the present study. These

427 findings are in general agreement with previous work using isometric contractions to characterize

428 the EMG force relationship. Studies assessing the relationship between force and EMG have

429 shown that as workload increases, EMG also increases both linearly (Lippold 1952; Woods \&

430 Bigland-Ritchie 1983) and non-linearly (Woods \& Bigland-Ritchie 1983). Importantly, Moritani

431 et al. (1978) reported a linear relationship between workload and EMG in the right elbow flexor

432 muscles during several submaximal contractions.

433

434 Gain of the iEMG-power output relationship during elbow flexion and extension phases 
436 different between elbow flexion and extension for each muscle with the exception of ECR ( $p=$

437 0.07). The most interesting finding here is that the triceps brachii 'gain' was higher during elbow

438 extension than flexion (i.e. as the power output increased there was a greater increase in iEMG

439 amplitude during elbow extension than flexion). This may relate to our thought that the triceps

440 brachii is active during flexion at lower intensities as a hand stabilizer and that as the intensity of

441 arm cycling increases the triceps brachii is recruited to produce elbow extension forces to a greater

442 degree to assist with arm crank movement.

443

444 Methodological Considerations

445 There are several methodological considerations to be taken considered when interpreting

446 the present results. The data collected in this study occurred over a relatively brief period (only 20

447 seconds). As the duration of sustained exercise increases, so too does the amplitude of EMG

448 signals (Takaishi et al. 1996). Had the trials been longer in the present study, the observed muscle

449 activity patterns may have been different. It is also important to note the difference in cycling

450 cadence between the maximal ergometry sprint and the cycling trials using different percentages

451 of PPO. During the maximal sprint, participants cycled as fast as they could against a set resistance

452 whereas a 60 rpm cadence was used during all submaximal trials in which EMG was assessed.

453 This likely lead to a much higher level of muscle activity during the cycling sprint than could be

454 obtained while cycling at a slower cadence due to the added influence of cadence-dependent

455 changes in descending drive and/or afferent feedback. This is an important consideration because

456 the cycling trials are normalized to the maximal sprint EMG, which partially explains the relatively

457 low level of EMG recorded from the muscles during the relative workloads, even at 50\% PPO. It 
458 is also important to recognize that arm cycling is a complex motor output that involves the 459 activation of many muscles working at several joints, bilaterally; at higher PPO intensities, 460 contributions from the torso and lower limbs are likely to be significant as well (Smith et al. 2008).

461 This means that the timing and co-ordination of muscle activity and/or muscle synergies are likely 462 of great importance when considering how the neuromuscular system produces this motor output, 463 particularly as power output increases (Blake \& Wakeling 2015; Enders et al. 2015; Wakeling et 464 al. 2010). We did not assess timing or synergies in the present study as our goal was to characterize 465 flexion/extension phase-dependent EMG amplitudes as power output increased given our interest 466 in how cycling intensity alters corticospinal excitability, which is dependent on the amount of 467 EMG activity produced. Also, the present study only examined muscle activity in two distinct 468 phases of arm cycling-flexion and extension relative to elbow joint movement. While this was 469 ultimately central to one of our main objectives, arm cycling can be characterized by more than 470 just two phases. Indeed, as can be seen in Figure 2, the muscle activity patterns of some muscles 471 change constantly throughout a full revolution. Had data been broken down into a greater number 472 of phases, a more complex phase-dependent behaviour would likely have been observed for all 473 examined muscles.

474 Lastly, two different ergometers (Monark and SCIFIT) were utilized in this study to fulfill 475 two separate needs (establish PPO and set stable target power outputs). These two needs could not 476 be addressed by one device alone. It is therefore possible that the power output measurement 477 precision between these devices was slightly different. If so, the relative targets used in this study 478 (5-50\% of PPO) may not have been precisely as stated. However, such differences were almost 479 certainly small and were unlikely to have influenced the main outcomes of this study. 


\section{Conclusion}

482

The present study characterized the iEMG pattern of activation during arm cycling at 483 different relative intensities. One of the main findings was a linear relationship between iEMG 484 amplitude and power output during arm cycling. We also showed that the influence of the 485 flexion/extension cycling phases were muscle-dependent (i.e. muscle activity differed between 486 these two phases in some muscles but not in others). Given the well-known impact of EMG 487 amplitude on various measures of neural excitability it may be of importance for individuals to 488 cycle at relative intensities (i.e. percentages of their maximal PPO).

489

490

\section{Acknowledgements}

491 This work was supported by a Discovery Grant to Kevin Power from the Natural Sciences 492 and Engineering Research Council of Canada (NSERC: \#RGPIN-2016-03646). The authors thank

493 Michael Monks for assistance with data collection, Dr. Tim Alkanani for technical support and all 494 of the volunteer participants.

495 
496

497

498

499

500

501

502

503

504

505

506

507

508

509

510

511

512

513

514

515

516

517

518

519

520

521

522

523

524

525

526

527

528

529

530

531

532

533

534

535

536

\section{References}

Adrian ED, and Bronk DW. 1929. THE DISCHARGE OF IMPULSES IN MOTOR NERVE FIBRES. Part II. The frequency of discharge in reflex and voluntary contractions. Journal of PhysiologyLondon 67:119-151.

Aoki F, Nagasaki H, and Nakamura R. 1986. The relation of integrated EMG of the triceps brachii to force in rapid elbow extension. Tohoku J Exp Med 149:287-291.

Barnes WS. 1980. The relationship of Motor-unit activation to isokinetic muscular contraction at different contractile velocities. Phys Ther 60:1152-1158.

Bernasconi S, Tordi N, Perrey S, Parratte B, and Monnier G. 2006. Is the VO2 slow component in heavy arm-cranking exercise associated with recruitment of type II muscle fibers as assessed by an increase in surface EMG? Appl Physiol Nutr Metab 31:414-422. 10.1139/h06-021

Bigland-Ritchie B, and Woods JJ. 1974. Integrated EMG and oxygen uptake during dynamic contractions of human muscles. J Appl Physiol 36:475-479.

Blake OM, and Wakeling JM. 2015. Muscle Coordination Limits Efficiency and Power Output of Human Limb Movement under a Wide Range of Mechanical Demands. J Neurophysiol:jn 00765 02015. 10.1152/jn.00765.2015

Bressel E, Bressel M, Marquez M, and Heise GD. 2001. The effect of handgrip position on upper extremity neuromuscular responses to arm cranking exercise. J Electromyogr Kinesiol 11:291-298.

Brousseau J, Malik, R., Chisholm, A.E., Lynn, A., Williams, A., Lam, T. . 2016. Can arm cycle training effect postural control and voluntary trunk muscle activation in people with spinal cord injury? . Society for Neuroscience. San Diego, California.

Carroll TJ, Baldwin ER, Collins DF, and Zehr EP. 2006. Corticospinal excitability is lower during rhythmic arm movement than during tonic contraction. J Neurophysiol 95:914-921.

Copithorne DB, Forman DA, and Power KE. 2015. Premovement Changes in Corticospinal Excitability of the Biceps Brachii are Not Different Between Arm Cycling and an IntensityMatched Tonic Contraction. Motor Control 19:223-241. 10.1123/mc.2014-0022

Duchateau J, Le Bozec S, and Hainaut K. 1986. Contributions of slow and fast muscles of triceps surae to a cyclic movement. Eur J Appl Physiol Occup Physiol 55:476-481.

Enders H, V VONT, and Nigg BM. 2015. Neuromuscular Strategies during Cycling at Different Muscular Demands. Med Sci Sports Exerc 47:1450-1459. 10.1249/MSS.0000000000000564

Farina D. 2006. Interpretation of the surface electromyogram in dynamic contractions. Exerc Sport Sci Rev 34:121-127.

Forman D, Raj A, Button DC, and Power KE. 2014. Corticospinal excitability of the biceps brachii is higher during arm cycling than an intensity-matched tonic contraction. J Neurophysiol 112:1142-1151. 10.1152/jn.00210.2014

Forman DA, Monks M, and Power KE. 2019. Corticospinal excitability, assessed through stimulus response curves, is phase-, task-, and muscle-dependent during arm cycling. Neurosci Lett 692:100-106. 10.1016/j.neulet.2018.11.003 
537 Forman DA, Philpott DT, Button DC, and Power KE. 2015. Cadence-dependent changes in

538

539

540

541

542

543

544

545

546

547

548

549

550

551

552

553

554

555

556

557

558

559

560

561

562

563

564

565

566

567

568

569

570

571

572

573

574

575

576

577

578

579 corticospinal excitability of the biceps brachii during arm cycling. J Neurophysiol 114:2285-2294. 10.1152/jn.00418.2015

Forman DA, Philpott DT, Button DC, and Power KE. 2016a. Differences in corticospinal excitability to the biceps brachii between arm cycling and tonic contraction are not evident at the immediate onset of movement. Experimental Brain Research 234:23392349. 10.1007/s00221-016-4639-z

Forman DA, Richards M, Forman GN, Holmes MW, and Power KE. 2016b. Changes in Corticospinal and Spinal Excitability to the Biceps Brachii with a Neutral vs. Pronated Handgrip Position Differ between Arm Cycling and Tonic Elbow Flexion. Front Hum Neurosci 10:543. 10.3389/fnhum.2016.00543

Henneman E. 1957. Relation between size of neurons and their susceptibility to discharge. Science 126:1345-1347.

Hug F, Laplaud D, Lucia A, and Grelot L. 2006. EMG threshold determination in eight lower limb muscles during cycling exercise: a pilot study. Int J Sports Med 27:456-462. 10.1055/s2005-865787

Hug F, Laplaud D, Savin B, and Grelot L. 2003. Occurrence of electromyographic and ventilatory thresholds in professional road cyclists. Eur J Appl Physiol 90:643-646. 10.1007/s00421003-0949-5

Hundza SR, de Ruiter GC, Klimstra M, and Zehr EP. 2012. Effect of afferent feedback and central motor commands on soleus H-reflex suppression during arm cycling. J Neurophysiol 108:3049-3058. 10.1152/jn.00485.2011

Kaupp C, Pearcey GEP, Klarner T, Sun Y, Cullen H, Barss TS, and Zehr EP. 2018. Rhythmic arm cycling training improves walking and neurophysiological integrity in chronic stroke: the arms can give legs a helping hand in rehabilitation. Journal of Neurophysiology 119:1095-1112. 10.1152/jn.00570.2017

Klarner T, Barss TS, Sun Y, Kaupp C, Loadman PM, and Zehr EP. 2016. Long-Term Plasticity in Reflex Excitability Induced by Five Weeks of Arm and Leg Cycling Training after Stroke. Brain Sciences 6. 10.3390/brainsci6040054

Klimstra MD, Thomas E, and Zehr EP. 2011. Biomechanical outcomes and neural correlates of cutaneous reflexes evoked during rhythmic arm cycling. J Biomech 44:802-809. 10.1016/j.jbiomech.2010.12.017

Lippold OC. 1952. The relation between integrated action potentials in a human muscle and its isometric tension. J Physiol 117:492-499.

Lucia A, Vaquero AF, Perez M, Sanchez O, Sanchez V, Gomez MA, and Chicharro JL. 1997. Electromyographic response to exercise in cardiac transplant patients: a new method for anaerobic threshold determination? Chest 111:1571-1576.

Nippard AP, Lockyer EJ, Button DC, and Power KE. 2020. Corticospinal excitability to the biceps and triceps brachii during forward and backward arm cycling is direction- and phasedependent. Appl Physiol Nutr Metab 45:72-80. 10.1139/apnm-2019-0043

Pearcey GE, Power KE, and Button DC. 2014. Differences in Supraspinal and Spinal Excitability during Various Force Outputs of the Biceps Brachii in Chronic- and Non-Resistance Trained Individuals. Plos One 9:e98468. 10.1371/journal.pone.0098468 
580

581

582

583

584

585

586

587

588

589

590

591

592

593

594

595

596

597

598

599

600

601

602

603

604

605

606

607

608

609

610

611

612

613

614

615
Power KE, and Copithorne DB. 2013. Increased corticospinal excitability prior to arm cycling is due to enhanced supraspinal but not spinal motoneurone excitability. Appl Physiol Nutr Metab 38:1154-1161. 10.1139/apnm-2013-0084

Power KE, Lockyer EJ, Forman DA, and Button DC. 2018. Modulation of motoneurone excitability during rhythmic motor outputs. Appl Physiol Nutr Metab:1-10. 10.1139/apnm-2018-0077

Sidhu SK, Hoffman BW, Cresswell AG, and Carroll TJ. 2012. Corticospinal contributions to lower limb muscle activity during cycling in humans. J Neurophysiol 107:306-314. 10.1152/jn.00212.2011

Smith PM, Chapman ML, Hazlehurst KE, and Goss-Sampson MA. 2008. The influence of crank configuration on muscle activity and torque production during arm crank ergometry. $J$ Electromyogr Kinesiol 18:598-605. 10.1016/j.jelekin.2006.12.006

Spence AJ, Alcock LR, Lockyer EJ, Button DC, and Power KE. 2016. Phase- and WorkloadDependent Changes in Corticospinal Excitability to the Biceps and Triceps Brachii during Arm Cycling. Brain Sciences 6. 10.3390/brainsci6040060

Takaishi T, Yasuda Y, Ono T, and Moritani T. 1996. Optimal pedaling rate estimated from neuromuscular fatigue for cyclists. Med Sci Sports Exerc 28:1492-1497. 10.1097/00005768-199612000-00008

Taylor AD, and Bronks R. 1994. Electromyographic correlates of the transition from aerobic to anaerobic metabolism in treadmill running. Eur J Appl Physiol Occup Physiol 69:508-515.

Wakeling JM, Blake OM, and Chan HK. 2010. Muscle coordination is key to the power output and mechanical efficiency of limb movements. J Exp Biol 213:487-492. $10.1242 /$ jeb. 036236

Woods JJ, and Bigland-Ritchie B. 1983. Linear and non-linear surface EMG/force relationships in human muscles. An anatomical/functional argument for the existence of both. Am J Phys Med 62:287-299.

Zehr EP, Carroll TJ, Chua R, Collins DF, Frigon A, Haridas C, Hundza SR, and Thompson AK. 2004. Possible contributions of CPG activity to the control of rhythmic human arm movement. Can J Physiol Pharmacol 82:556-568.

Zehr EP, and Chua R. 2000. Modulation of human cutaneous reflexes during rhythmic cyclical arm movement. Experimental Brain Research 135:241-250.

Zehr EP, and Duysens J. 2004. Regulation of arm and leg movement during human locomotion. Neuroscientist 10:347-361. 
616 Figure Legends

617

618 Figure 1. Example of the experimental setup. Participants were seated with their shoulders at 619 approximately the same height as the axis of the crank shaft on the SCIFIT cycle ergometer while 620 cycling at a constant cadence of $60 \mathrm{rpm}$ at 11 different workloads $(5-50 \%$ of PPO and $25 \mathrm{~W})$.

621 Flexion and extension were made relative to the elbow joint (flexion from 3 to 9 o'clock and 622 extension from 9 to 3 o'clock; both relative to a clock face; arm in the example at the 6 o'clock 623 position). iEMG was recorded from biceps brachii, triceps brachii, anterior deltoid, brachioradialis, 624 FCR and ECR from the dominant limb.

625

626 Figure 2. Linear envelope ensemble averaged iEMG during 1 full revolution for each muscle 627 examined. Arm cycling intensities shown include the absolute power output (25W) and the 628 percentages of maximum PPO $(10,20,30,40$ and 50\%). The remaining intensities of arm cycling 629 are excluded for figure clarity $(5,15,25,35$ and 45\% PPO). Amplitudes are expressed as a 630 percentage of maximal EMG. The dark gray region in each section indicates elbow flexion (3 to 9 631 o'clock) while the light gray region represents elbow extension (9 to 3 o'clock).

632

633 Figure 3. The relationship between iEMG and power output for each muscle during the elbow 634 flexion and extension phases of arm cycling. The slopes and $r$ values are illustrated for each 635 position in each muscle. Slopes were significantly different for muscles shown in Figs. A-D and 636 F. While the slopes were not significantly different between flexion and extension in ECR $(\mathrm{p}=$ 6370.07 ; Fig. 3F), they are plotted to allow visual inspection. 


\section{Table 1 (on next page)}

iEMG and workload. Statistical summary table. 
1 Table 1. iEMG and workload. Statistical summary table.

2

\begin{tabular}{|c|c|c|c|}
\hline Muscle & $\begin{array}{c}\text { Position } \\
\text { Main Effect }\end{array}$ & $\begin{array}{c}\text { Intensity } \\
\text { Main Effect }\end{array}$ & $\begin{array}{l}\text { Interaction } \\
\text { Main Effect }\end{array}$ \\
\hline Biceps Brachii & $\begin{array}{l}\left(F_{(1,10)}=105.363, p<\right. \\
.001)\end{array}$ & $\begin{array}{l}\left(F_{(2.72,27.22)}=59.435,\right. \\
p<.001)\end{array}$ & $\begin{array}{l}\left(F_{(2.98,29.18)}=41.737,\right. \\
p<.001)\end{array}$ \\
\hline Triceps Brachii & $\begin{array}{l}{\left(F_{(1,10)}=1.362, p=\right.}_{.270)}\end{array}$ & $\begin{array}{l}\left(F_{(2.06,20.6)}=65.015,<\right. \\
.001)\end{array}$ & $\begin{array}{l}{\left(F_{(1.51,15.16)}=2.246, p\right.} \\
=.148)\end{array}$ \\
\hline Anterior Deltoid & $\begin{array}{l}{\left(F_{(1,4)}=17.067, p=\right.}_{.014)}\end{array}$ & $\begin{array}{l}\left(F_{(10,40)}=15.110, p=\right. \\
.001)\end{array}$ & $\begin{array}{l}{\left(F_{(10,40)}\right.}=15.039, p= \\
.001)\end{array}$ \\
\hline Brachioradialis & $\begin{array}{l}{\left(F_{(1,4)}=37.097, p=\right.}_{.004)}\end{array}$ & $\begin{array}{l}\left(F_{(10,40)}=37.954, p<\right. \\
.001)\end{array}$ & $\begin{array}{l}{\left(F_{(10,40)}=28.886, p<\right.}< \\
.001)\end{array}$ \\
\hline FCR & $\begin{array}{l}\left(F_{(1,4)}=10.484, p=\right. \\
.032)\end{array}$ & $\begin{array}{l}\left(F_{(10,40)}=56.171, p<\right. \\
.001)\end{array}$ & $\begin{array}{l}\left(_{(10,40)}=4.772, p=\right. \\
.040)\end{array}$ \\
\hline ECR & $\begin{array}{l}{\left(F_{(1,4)}=.217, p=\right.}_{.665)}\end{array}$ & $\begin{array}{l}\left(F_{(10,40)}=27.585, p<\right. \\
.001)\end{array}$ & $\begin{array}{l}{\left(F_{(10,40)}=.509, p=\right.}= \\
.579)\end{array}$ \\
\hline
\end{tabular}

3

4

5

6

7 
Table 2 (on next page)

Relationships between iEMG and workload. Statistical summary table. 
1 Table 2. Relationships between iEMG and workload. Statistical summary table.

\begin{tabular}{|c|c|c|c|c|}
\hline Muscle & Phase & Linear & Quadratic & Cubic \\
\hline Biceps Brachii & $\mathrm{F}$ & $102.766_{(1,10),} p<.001$ & $3.138_{(1,10)}, p=.107$ & $\begin{array}{l}4.669_{(1,10),} \mathrm{p}= \\
.056\end{array}$ \\
\hline Biceps Brachii & $\mathrm{E}$ & $83.347_{(1,10),} \mathrm{p}<.001$ & $.541_{(1,10),} \mathrm{p}=.479$ & $\begin{array}{l}10.114_{(1,10),} \mathrm{p}= \\
.010^{*}\end{array}$ \\
\hline Triceps Brachii & $\bar{F}$ & $41.255_{(1,10),} \mathrm{p}<.001$ & $2.047_{(1,10),} p=.183^{2}$ & $\begin{array}{l}1.257_{(1,10),} \mathrm{p}= \\
.288\end{array}$ \\
\hline Triceps Brachii & $\mathrm{E}$ & $85.090_{(1,10),} \mathrm{p}<.001$ & $3.653_{(1,10),} \mathrm{p}=.085$ & $\begin{array}{l}3.764_{(1,10),} \mathrm{p}= \\
.081\end{array}$ \\
\hline Anterior Deltoid & $\mathrm{F}$ & $20.378_{(1,4),} \mathrm{p}<.05$ & $.172_{(1,4),} p=.700$ & $\begin{array}{l}3.681_{(1,4),} \mathrm{p}= \\
.127\end{array}$ \\
\hline Anterior Deltoid & $\mathrm{E}$ & $28.356_{(1,4),} \mathrm{p}<.01$ & $.222_{(1,4), p}=.662$ & $\begin{array}{l}12.342_{(1,4),} \mathrm{p}= \\
.025\end{array}$ \\
\hline Brachioradialis & $\mathrm{F}$ & $85.296_{(1,4),} p=.001$ & $7.819_{(1,4),} p=.049$ & $\begin{array}{l}1.760_{(1,4),} \mathrm{p}= \\
.255\end{array}$ \\
\hline Brachioradialis & $\mathrm{E}$ & $40.878_{(1,4),} \mathrm{p}<.01$ & $6.775_{(1,4), p}=.060$ & $\begin{array}{l}23.252_{(1,4),} \mathrm{p}= \\
.009\end{array}$ \\
\hline FCR & $\bar{F}$ & $449.438_{(1,4)}, \mathrm{p}<.001$ & $5.663_{(1,4),} \mathrm{p}=.076$ & $\begin{array}{l}1.584_{(1,4),} \mathrm{p}= \\
.277\end{array}$ \\
\hline FCR & $\mathrm{E}$ & $25.641_{(1,4),} \mathrm{p}<.01$ & $.000_{(1,4),} p=.995$ & $\begin{array}{l}.573_{(1,4),} \mathrm{p}= \\
.491\end{array}$ \\
\hline ECR & $\mathrm{F}$ & $49.141_{(1,4),} \mathrm{p}<.01$ & $2.233_{(1,4),} p=.209$ & $\begin{array}{l}1.236_{(1,4),} \mathrm{p}= \\
.329\end{array}$ \\
\hline ECR & $\mathrm{E}$ & $19.137_{(1,4),}, p<.05$ & $2.342_{(1,4),} p=.201$ & $1.654_{(1,4),} p=$ \\
\hline
\end{tabular}




\begin{tabular}{|l|l|l|l|l|}
\hline & & & & .268 \\
\hline
\end{tabular}

2 Note: $\mathrm{n}=11$ for biceps and triceps brachii and $\mathrm{n}=5$ for the remaining muscles. $\mathrm{F}=$ flexion phase

3 and $\mathrm{E}=$ extension phase.

4 


\section{Figure 1}

Example of the experimental setup

Participants were seated with their shoulders at approximately the same height as the axis of the crank shaft on the SCIFIT cycle ergometer while cycling at a constant cadence of $60 \mathrm{rpm}$ at 11 different workloads (5-50\% of PPO and $25 \mathrm{~W}$ ). Flexion and extension were made relative to the elbow joint (flexion from 3 to 9 o'clock and extension from 9 to 3 o'clock; both relative to a clock face; arm in the example at the 6 o'clock position). iEMG was recorded from biceps brachii, triceps brachii, anterior deltoid, brachioradialis, FCR and ECR from the dominant limb. 


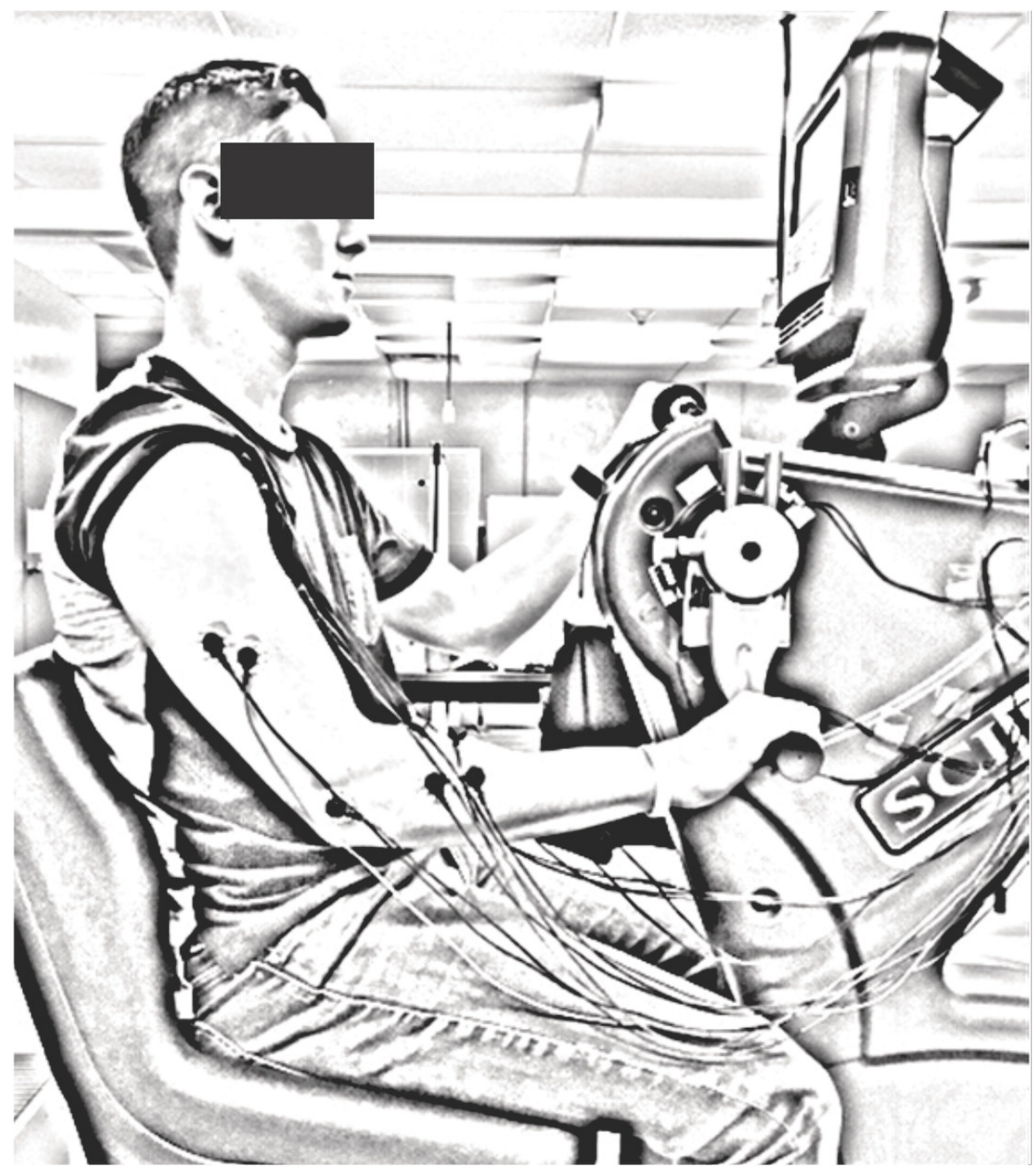




\section{Figure 2}

Linear envelope ensemble averaged iEMG during 1 full revolution for each muscle examined

Arm cycling intensities shown include the absolute power output (25W) and the percentages of maximum PPO (10, 20, 30, 40 and 50\%). The remaining intensities of arm cycling are excluded for figure clarity $(5,15,25,35$ and $45 \%$ PPO). Amplitudes are expressed as a percentage of maximal EMG. The dark gray region in each section indicates elbow flexion (3 to $9 o^{\prime}$ clock) while the light gray region represents elbow extension (9 to 3 o'clock). 

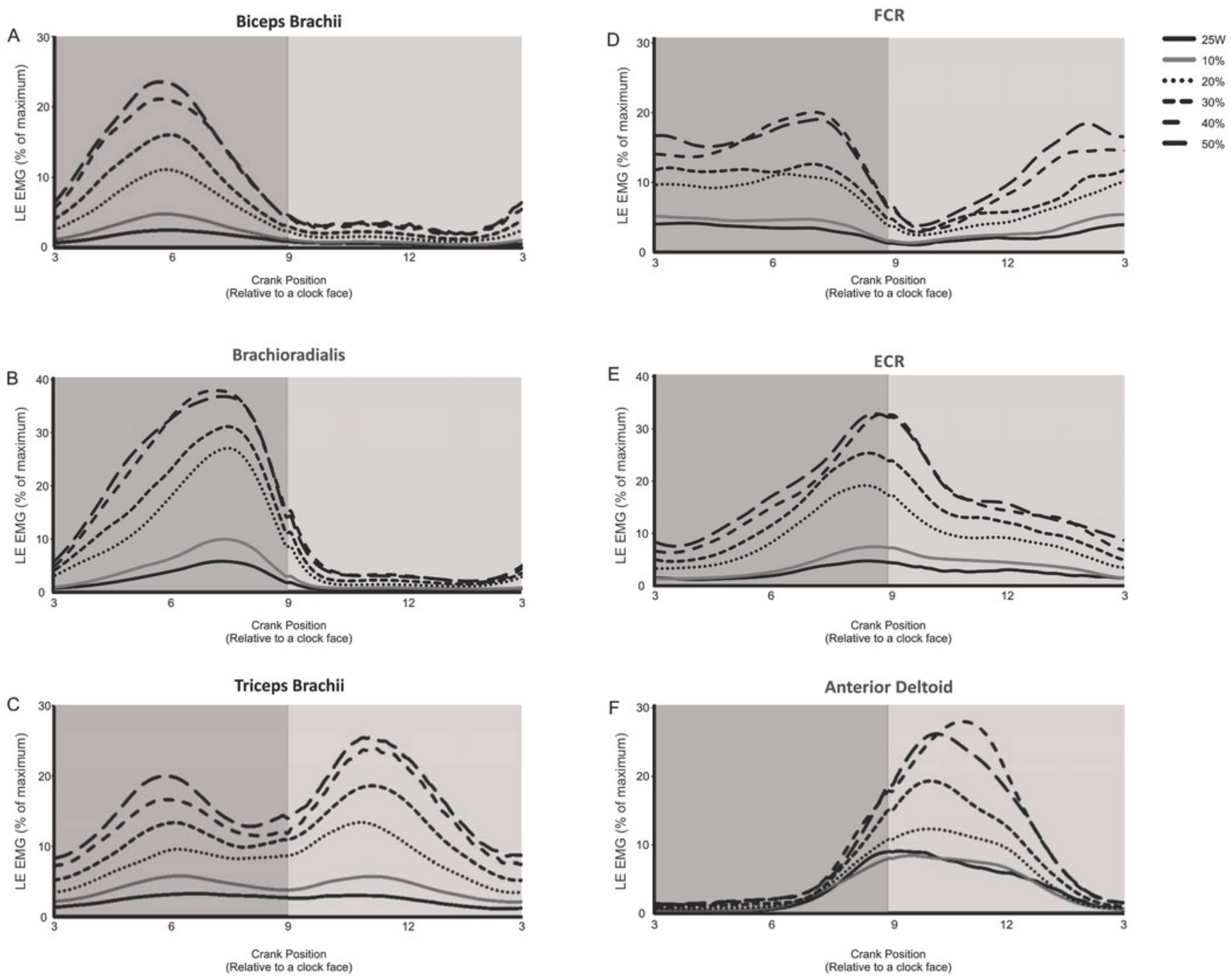

Elbow Flexion

Elbow Extension 
Figure 3

The relationship between iEMG and power output for each muscle during the elbow flexion and extension phases of arm cycling

The slopes and $r$ values are illustrated for each position in each muscle. Slopes were significantly different for muscles shown in Figs. A-D and F. While the slopes were not significantly different between flexion and extension in ECR ( $p=0.07$; Fig. 3F), they are plotted to allow visual inspection. 
A

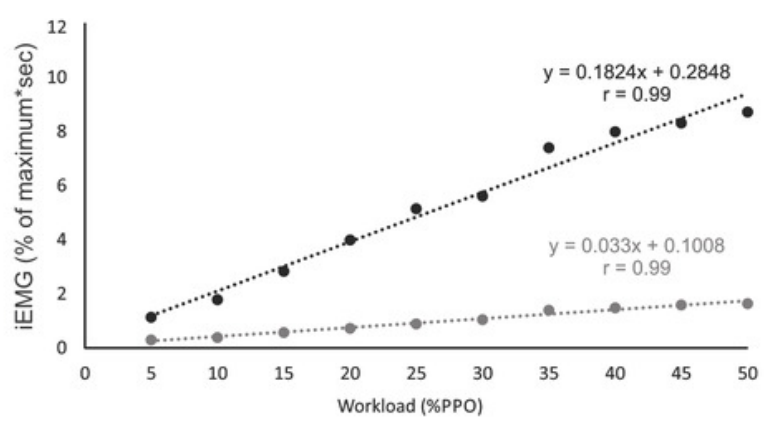

B

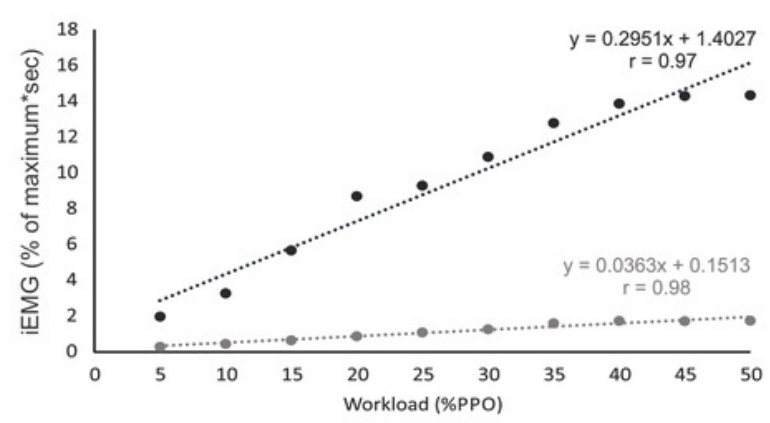

C

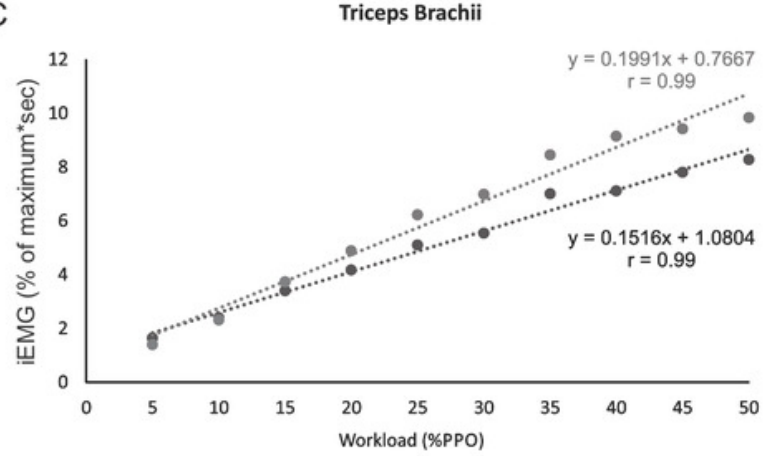

D

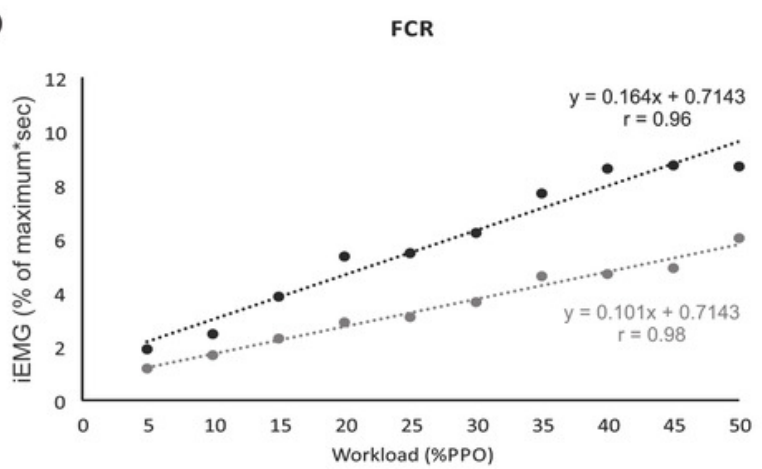

E

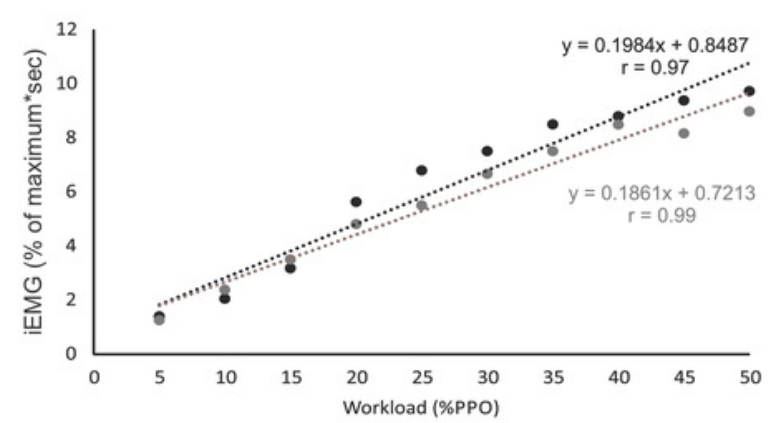

F

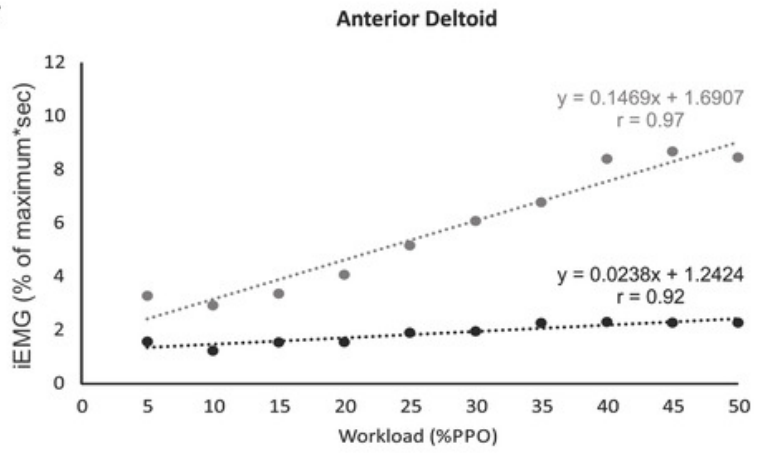

